\title{
Probabilistic assessment of the role of the soil degradation main factors in Kuban rice fields
}

\author{
Tatyana Safronova ${ }^{1}$, Stanislav Vladimirov ${ }^{1}$ and Igor Prikhodko ${ }^{1, *}$ \\ ${ }^{1}$ Kuban State Agrarian University named after I. T. Trubilin, 13, Kalinina str., 350044, Krasnodar, \\ Russia
}

\begin{abstract}
As a result of reclamation systems for rice cultivation construction, the conditions of genesis and landscapes soil formation were radically violated. This led to the leaching of active humus and calcium, colloidal particles, nutrients from arable layer to underlying horizons, as a result of which there is a tendency to secondary salinization and solonization of soils. The development of the eluvial glue process and physicochemical parameters change of soils causes a change in the morphological soil profile. Currently relevant are preventing land degradation problems, maintaining and restoring soil fertility. In the article, the authors propose to characterize the rice irrigation system functioning mode from probabilistic point of view. They offer to evaluate the consequences of anthropogenic load, considering operational activities by Poisson stream of a certain intensity. This approach allows to consider uncertainty in terms of probability distributions. The function, characterizing soil quality $S(t)$ was introduced. The function $S(t)$ assumed to be monotonously decreasing. The probability $R(S)$ of achieving a certain soil quality was considered. In case of minimum amount of humus $S_{m}$ the soil is degrading. In these assumptions the average value expression and probability density of particular soil condition onset duration was received.
\end{abstract}

\section{Introduction}

In historical aspect a significant transformation of Lower Kuban landscapes has been occurred. Especially landscapes in the delta-flooded zone of the Kuban river were exposed to this process.

Such transition is caused by a change of redox balance of soil reactions and redistribution of surface and ground runoff volume ratio [1-3].

As a result, soil restoration processes, flowing in swamp type, acquired a seasonal (shift) character. The fundamentally new approach of soils redox potential regulation, as the basis of technological processes in rice cultivation as a reclamation crop was laid. Main focus in technological presowing operational tillage was aimed at stimulating oxidative processes $[4,5]$.

Consequently - natural delta landscapes largely unsuitable for agricultural crops cultivation acquired properties of cultural irrigation and rice agrolandscapes $[6,7]$.

\footnotetext{
* Corresponding author: prihodkoigor2012@yandex.ru
} 
These properties strengthened engineering systems, accomplished in ameliorative aspect, in which the flushing mode was provided, water supply and drainage were divided, and regulation of ground runoff level mode formation conditions was carried out [8].

As a result of providing such more than 50 years of soil regime, so-called «rice fields» were formed: meadow chernozem, meadow, alluvial meadow saturated and alluvial meadow-swamp [9].

After such historical transformation the soils received new consumer properties, social and environmental importance.

However anthropogenic impacts to such system have similar trends, specific for irrigation agriculture. Relevant in this case are problems of land degradation preventing, maintaining and restoring soil fertility $[10,11]$.

Based on the foregoing provisions, the new rice cultivation concept on Kuban can be represented by main blocks - ecosystem components (anthropogenic activities, soils, plant, water).

The «soil» block main aspects are: soils physical condition restoration and protection of soils from physical degradation; soils humus state restoration and its protection; sustainable land use; soil protection against erosion and pesticide pollution, as well as other events, aimed at environmentally sound agrolandscapes creating, which would reduce or completely eliminate the agrochemicals migration flows [12].

The «plant» block resolves problems of farmland structures ecologization, crop rotation, immune varieties introduction, development and implementation of organic and organic mineral ecologically fertilizer systems and organic fertilizer production technologies, development and implementation environmentally friendly weed control systems, diseases and crop pests [13].

The «water» block is aimed to resolve problems of surface and ground water regulation, accumulation and pollution protection, its rational use in agricultural production without disturbances in the region ecosystem, and Azov and Black Seas environmental improvement.

The «anthropogenic activities» block is aimed to solve issues of agrochemical services improvement in terms of ecologization and improving of legal and economic agroecosystems protection mechanism from environmental disruptions as a result of agricultural activities.

\section{Material and methods of the study}

Considering Kuban rice fields soil degradation main factors from a probabilistic point of view - humus reduction, quality deterioration, soil upper horizons decalcification and alkalization $[14,15]$.

Accepting $S(t)$ - specific soil property (for example, the presence of a certain quantity and humus property). Considering $S(t)$ as monotonically decreasing function by time from some value $S_{0}=S(0)$. The equation $S(t)=S$ can be explicitly resolved with respect to the argument $t$ and get the ratio $t=t(S)$.

To assess the consequences of anthropogenic load the intensity of impact on the soil of machines and mechanisms should be considered. It should be noted, that rice-planting farms at currents time owns the technique by total power more than $250 \mathrm{~h}$. p. for 100 ha irrigated area. For 1 ha of rice sown area an amount of more than 200 reference ha of tractor works is carried out and from 16 to 25 thousand $\mathrm{m}^{3}$ irrigation water is serving. Modern tractors, especially wheeled, put pressure onto the soil till $3-4 \mathrm{~kg} / \mathrm{sm}^{2}$ and more, and ground drive systems cover the area of the field till $80 \%$ only during pre-sowing and sowing period [16]. 
We assume that ongoing reclamation activities form a Poisson stream by constant intensity $\lambda$. We introduce the notation: $R(S)$ - probability of achieving soil quality, determined by quality. Then $1-R(S)$ is a probability that the soil does not have right quality. Further we assume that $R(S)$ - monotonically decreasing function, i.e. the soil quality is decreasing with time. Besides, we assume, that with a minimum amount of humus $S_{m}$ soil degradates, so $R\left(S_{m}\right)=1$ and $\lim _{t \rightarrow \infty} S(t)=S_{m}$.

\section{Results}

We will prepare a probabilistic characteristic (mathematical expectation) of certain soil condition onset duration.

Time $\tau$ between the timepoint $t$ and the moment when soil acquired a certain quality $-\mathrm{a}$ random value. We denote $m_{t}(S)$ conditional mathematical expectation of $\tau$ value in term that at the timepoint $t$ soil did not have the considering quality and its characteristic was equal $S$, i.e. $m_{t}(S)=M\{\tau \mid S(t)=S\}$. We compose the equation for $m_{t}(S)$.

For timepoint $t+\Delta t$ it can be noted, that for the time interval $\Delta t$ with probability $\lambda R(S) \Delta t$ a certain condition will come and the remaining time will become equal 0 . With probability $1-\lambda R(S) \Delta t$ condition will not be reached and the remaining time will become equal $m_{t}(S+\Delta S)$. Given the above reasoning, we can write

$$
m_{t}(S)=\Delta t+\lambda R(S) \Delta t \cdot 0+(1-\lambda R(S) \Delta t) m_{t}(S+\Delta S)
$$

For $m_{t}(S+\Delta S)$

$$
m_{t}(S)=\Delta t+(1-\lambda R(S) \Delta t)\left[m_{t}(S)+m^{\prime}{ }_{t}(S) \cdot \Delta S\right] .
$$

After transformations and transition to the limit at $\Delta t \rightarrow 0$ we get

$$
0=1-\lambda R(S) m_{t}(S)+m^{\prime}{ }_{t}(S) \frac{d S}{d t}
$$

We find the addiction $t=t(S)$ and introduce the function

$$
a(S)=-\frac{d S}{d t} \mid t=t(S) .
$$

Since the function $S$ - decreasing, $a(S)>0$.

We denote $(S)=\lambda R(S) / a(S)$.

Meaning expression for function $(S)$, we can write

$$
m^{\prime}{ }_{t}(S) a(S)+\lambda p(S) m_{t}(S)=1,
$$

After dividing by $a(S)$ we finally obtain the equation for $m_{t}(S)$ :

$$
m_{t}^{\prime}(S)+g(S) m_{t}(S)=\frac{1}{a(S)} .
$$

General solution to the equation (3)

$$
m_{t}(S)=C_{0} \cdot \exp \left(-\int_{S_{m}}^{S} g(x) d x\right)+\int_{S_{m}}^{S} \frac{1}{a(y)} \exp \left(-\int_{y}^{S} g(x) d x\right) d y .
$$

To find an arbitrary constant, we will carry out the following reasoning. If $S(t)=S_{m}$ and $R\left(S_{m}\right)=1$, then we can confirm, that the average time is $1 / \lambda$, since the flow of events is a Poisson flow of intensity $\lambda,[17]$. And then from (4) at $S=S_{m}$ we get $C_{0}=1 / \lambda$. 


$$
m_{t}(S)=\frac{1}{\lambda} \cdot \exp \left(-\int_{S_{m}}^{S} g(x) d x\right)+\int_{S_{m}}^{S} \frac{1}{a(y)} \exp \left(-\int_{y}^{S} g(x) d x\right) d y
$$

Expression (5) determines the average duration of the onset of a certain soil condition.

Now we find a probability density of certain state offensive duration, using the Laplace transformation. We introduce the function

$$
G_{t}(q, S)=M\left\{e^{-q \tau} \mid S(t)=S\right\} .
$$

For the time point $t+\Delta t$ we can write

$$
G_{t}(q, S)=\lambda R(S) \Delta t \cdot e^{-q \Delta t}+\left(1-\lambda R(S) \Delta t \cdot e^{-q \Delta t} G_{t}(q, S+\Delta S)\right)
$$

Then we have

$$
\begin{gathered}
e^{-q \Delta t}=1-q \Delta t+o(\Delta t), \\
G_{t}(q, S+\Delta S)=G_{t}(q, S)+\frac{\partial G_{t}(q, S)}{\partial S} \Delta S+o(\Delta t) .
\end{gathered}
$$

Substitute prepared expressions into (7):

$$
G_{t}(q, S)=G_{t}(q, S)+\frac{\partial G_{t}(q, S)}{\partial S} \Delta S-(\lambda R(S)+q) G_{t}(q, S) \Delta t+\lambda R(S) \Delta t .
$$

After transformations and transition to the limit at $\Delta t \rightarrow 0$, find

$$
\frac{\partial G_{t}(q, S)}{\partial S} a(S)+(\lambda R(S)+q) G_{t}(q, S)=\lambda R(S),
$$

or

$$
\frac{\partial G_{t}(q, S)}{\partial S}+\left(g(S)+\frac{q}{a(S)}\right) G_{t}(q, S)=g(S) .
$$

We write the general solution of the corresponding homogeneous equation

$$
\begin{gathered}
\frac{\partial G_{t}(q, S)}{\partial S}+\left(g(S)+\frac{q}{a(S)}\right) G_{t}(q, S)=0 \\
G_{t}(q, S)=C(q) \cdot \exp \left(-\int_{S_{m}}^{S}\left(g(x)+\frac{q}{a(x)}\right) d x\right)
\end{gathered}
$$

Then we find a general solution to the equation (8) as

$$
G_{t}(q, S)=C(q, S) \cdot \exp \left(-\int_{S_{m}}^{S}\left(g(x)+\frac{q}{a(x)}\right) d x\right)
$$

Using the resulting expression, we can write

$$
\frac{\partial C(q, S)}{\partial S}=g(S) \cdot \exp \left(\int_{S_{m}}^{S}\left(g(x)+\frac{q}{a(x)}\right) d x\right),
$$

and get

$$
C(q, S)=C_{0}(q)+\int_{S_{m}}^{S} g(y) \cdot \exp \left(\int_{S_{m}}^{y}\left(g(x)+\frac{q}{a(x)}\right) d x\right) d y .
$$


Thus, the general solution of equation (8) has the form

$$
G_{t}(q, S)=C_{0}(q) \cdot \exp \left(-\int_{S_{m}}^{S}\left(g(x)+\frac{q}{a(x)}\right) d x\right)+\int_{S_{m}}^{S} g(y) \cdot \exp \left(-\int_{y}^{S}\left(g(x)+\frac{q}{a(x)}\right) d x\right) d y .
$$

Find $C_{0}(q)$. At $S(t)=S_{m}, p\left(S_{m}\right)=1$. Since the flow of events is a Poisson flow of intensity $\lambda$, then the time interval values probability density before a certain condition will be equal $p(\tau)=\lambda e^{-\lambda \tau}$ and the Laplace transformation of this function has the form $\frac{\lambda}{\lambda+q}$ [18].

From the general solution (9) for $S=S_{m}$ we get

$$
G_{t}(q, S)=\frac{\lambda}{\lambda+q} \cdot \exp \left(-\int_{S_{m}}^{S}\left(g(x)+\frac{q}{a(x)}\right) d x\right)+\int_{S_{m}}^{S} g(y) \cdot \exp \left(-\int_{y}^{S}\left(g(x)+\frac{q}{a(x)}\right) d x\right) d y .
$$

If at the time point $t=0, S(0)=S_{0}$, then

$$
G_{t}\left(q, S_{0}\right)=\frac{\lambda}{\lambda+q} \cdot \exp \left(-\int_{S_{m}}^{S_{0}}\left(g(x)+\frac{q}{a(x)}\right) d x\right)+\int_{S_{m}}^{S_{0}} g(y) \cdot \exp \left(-\int_{y}^{S_{0}}\left(g(x)+\frac{q}{a(x)}\right) d x\right) d y .
$$

Next, we find the inverse Laplace transform of this expression.

Omitting intermediate calculations, we finally obtain

$$
p(\tau)=\left\{\begin{array}{c}
-g(S(\tau)) \cdot \exp \left(-\int_{S(\tau)}^{S_{0}} g(x) d x\right) S^{\prime}(\tau), 0 \leq \tau<t\left(S_{m}\right) \\
\lambda e^{-\lambda\left(\tau-t\left(S_{m}\right)\right)} \cdot \exp \left(-\int_{S_{m}}^{S_{0}} g(x) d x\right), \tau \geq t\left(S_{m}\right) .
\end{array}\right.
$$

Next, we check for $p(\tau)$ fulfillment of the normalization condition.

$$
\int_{t\left(S_{m}\right)}^{\infty} \lambda e^{-\lambda\left(\tau-t\left(S_{m}\right)\right)} d \tau \cdot \exp \left(-\int_{S_{m}}^{S_{0}} g(x) d x\right)=\exp \left(-\int_{S_{m}}^{S_{0}} g(x) d x\right) .
$$

Then,

$$
\begin{gathered}
-\int_{0}^{t\left(S_{m}\right)} g(S(\tau)) \cdot \exp \left(-\int_{S(\tau)}^{S_{0}} g(x) d x\right) S^{\prime}(\tau) d \tau=\int_{S_{m}}^{S_{0}} g(y) \cdot \\
\exp \left(-\int_{y}^{S_{0}} g(x) d x\right) d y==\int_{S_{m}}^{S_{0}} d_{y} \cdot \exp \left(-\int_{y}^{S_{0}} g(x) d x\right)=\left.\exp \left(-\int_{y}^{S_{0}} g(x) d x\right)\right|_{S_{m}} ^{S_{0}}= \\
1-\exp \left(-\int_{S_{m}}^{S_{0}} g(x) d x\right) .
\end{gathered}
$$

This implies that $\int_{0}^{\infty} p(\tau) d \tau=1$, that is, the normalization condition is fulfilled.

\section{Discussion}

It should be noted, that in the rice crop rotation with $65 \%$ rice saturation, the aboveground mass annual growth composes 5-6 t/ha, undergrounf 2,5-3 t/ha, but it does not offset loss of soil organic matter even when chopping and plowing straw.

Degradation phenomena in rice field soils to some extent connected to mechanical and physical impacts during tillage. Capital and operational plans application, large amount of other groundworks during rice systems construction, leads to the movement and mixing of large soil masses. The resulting new arable horizon, usually, is significantly different from original humus horizon of virgin soils, and besides in the direction of organic matter and mineral nutrition elements content reducing.

Considering probability density graphical interpretation with different sets of parameters, determining the specific soil quality 
Let the dependence of soil quality on time take the form

$$
S(t)=S=S_{m}+\left(S_{0}-S_{m}\right) e^{-\alpha t},
$$

so that $S(0)=S_{0}$ and $\lim _{t \rightarrow \infty} S(t)=S_{m}$.

Then

$$
\frac{d S}{d t}=-\alpha\left(S_{0}-S_{m}\right) e^{-\alpha t}=-\alpha\left(S-S_{m}\right)
$$

so that $\alpha(S)=\alpha\left(S-S_{m}\right)$. Function $\alpha(S)$ was introduced by equality (2)

We consider $R(S)$ as

$$
R(S)=\frac{S_{M}-S}{S_{M}-S_{m}}
$$

so that $R\left(S_{M}\right)=0$ and $R\left(S_{m}\right)=1$. Value $S_{M}$ - maximum quality, when a damage to environment was not detected, $S_{m}$ - minimum quality, when a damage to the environment was always noted.

In this case

$$
g(S)=\frac{\lambda}{\alpha} \cdot \frac{S_{M}-S}{\left(S_{M}-S_{m}\right)\left(S-S_{m}\right)}
$$

We denote $\frac{\lambda}{\alpha}=\rho$ - dimensionless quantity.

Taking into account (13) expression for probability density $p(\tau)$ of random value (duration of the system certain state onset) can be written in the following way:

$$
\begin{gathered}
p(\tau)= \\
\frac{\rho}{S_{M}-S_{m}} \cdot \frac{\left(S_{M}-S_{m}\right)-\left(S_{0}-S_{m}\right) e^{-\alpha t}}{\left(S_{0}-S_{m}\right) e^{-\alpha t}} \times \alpha\left(S_{0}-S_{m}\right) e^{-\alpha t} \times \exp \left(-\rho \int_{S_{m}+\left(S_{0}-S_{m}\right) e^{-\alpha t}}^{S_{0}} \frac{S_{M}-x}{\left(x-S_{m}\right)\left(S_{M}-S_{m}\right)} d x\right),
\end{gathered}
$$

Passing to the dimensionless quantity $\zeta=\alpha \tau$. After a number of simplifications, we get:

$$
p(\zeta)=\rho\left(1-\Delta e^{-\zeta}\right) \exp \left(-\rho \int_{e^{-\zeta}}^{1} \frac{1-\Delta u}{u} d u\right),
$$

Having calculated the integral, we finally obtain

$$
p(\zeta)=\rho\left(1-\Delta e^{-\zeta}\right) \exp \left(-\rho \zeta+\rho \Delta\left(1-e^{-\zeta}\right)\right), \zeta \geq 0 .
$$

In expression (17) we used the notation of the parameter

$$
\Delta=\frac{S_{0}-S_{m}}{S_{M}-S_{m}}
$$

which varies within $0 \leq \Delta \leq 1$.

It should be noted, that $p(0)=\rho(1-\Delta)$ and $p(+\infty)=0$.

Graph examples $p(\zeta)$ with different sets of parameters $\Delta$ and $\rho$ are shown at the figures $1-2$. 


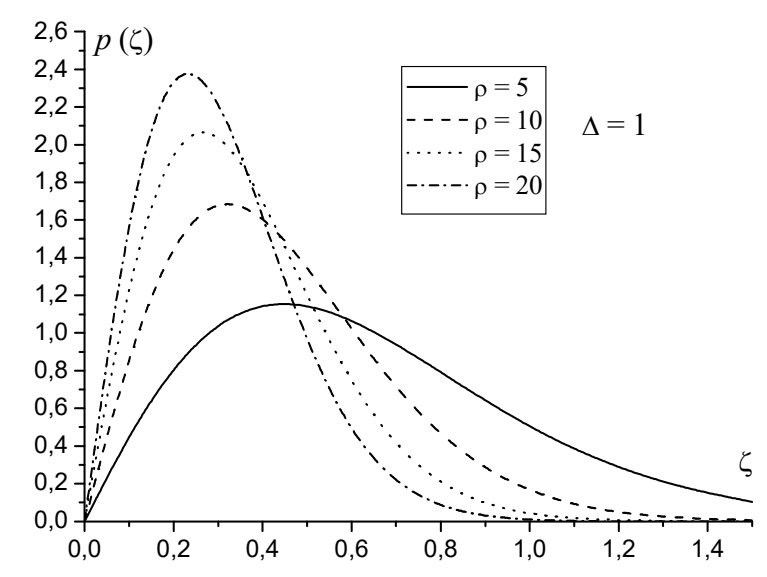

Fig. 1. Function graph $p(\zeta), \Delta=1$.

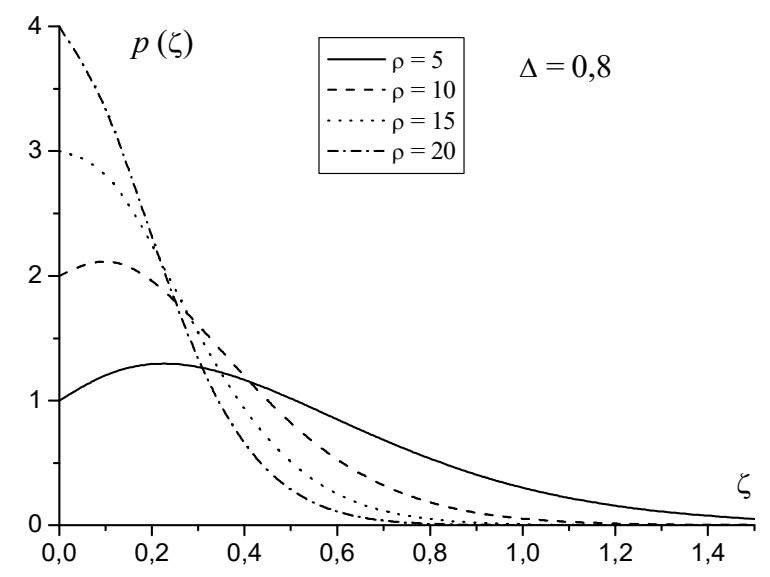

Fig. 2. Function graph $(\zeta), \Delta=0,8$.

\section{Conclusion}

The article suggests probabilistic assessment model of key factors of the Kuban rice fields' soil degradation role, which allows reducing uncertainty risks when making management decisions. It is proposed to consider the uncertainty in terms of probability distributions. Estimated parameters are treated by random variables. Operational activities are considered by a certain intensity Poisson flow in term of soil quality continuous change.

Mathematical model reflects the rice irrigation systems operating conditions, provides an assessment of the anthropogenic load effects and can expand taking into account all sorts of on soil assessment impact factors. Proposed can be used to control rice irrigation system depending on the climatic factors. Conducting preventive environmental measures will reduce capital costs on negative consequences elimination.

\section{References}

1. Nureize Arbaiy, Lin Pei-Chun, Lah Muhammad Shukri, Che Othman, Mohammad Haris Haikal, Advances in Intelligent Systems and Computing 978, 15-24 (2020) 
2. Kalinitchenko Valery P. Biogeosystem Technique, 4(10), 284-316 (2016)

3. R.A. Brain, M.F. Winchell, L.L. Richardson, K.A. Budreski, J. Bang, M.A. Feken, J.B. Dunne, Integrated Environmental Assessment and Management 15(6), 936-947 (2019)

4. T. Safronova, O. Degtyareva, S. Vladimirov, I. Prikhodko Research Journal of Pharmaceutical, Biological and Chemical Sciences 9(6), 1845-1852 (2018)

5. M.K. Shenk, D.A. Nolin, R.O. Begley, A. Swiatek, Philosophical Transactions of the Royal Society B: Biological Sciences 374(1780), 20190006 (2019)

6. O. Degtyareva, T. Safronova, I. Rudchenko, I. Prikhodko, IOP Conf. Series: Materials Science and Engineering 698, 022015 (2019) doi:10.1088/1757-899X/698/2/022015

7. S. Vladimirov, I. Prikhodko, A. Verbitskiy, Journal of Agriculture and Environment 1(9), 15 (2019)

8. E. Kuznetsov, T. Safronova, I. Sokolova, A. Khadzhidi, A. Gumbarov JEMT 81(17), 78-83 (2017)

9. A. Arthur, B. Barnes, Ecological Economics 161, 153-162 (2019)

10. C. Müller Felix, B. Burkhard, W. Windhorst, B. Dang Kinh, Ecological Indicators 100, 30-44 (2019)

11. L. Eike, D. Shepherd Keith, W. Cory William, D. Lanzanova, Environmental Modelling and Software 115, 164-175 (2019)

12. L. Jat Mangi, C. Sharma Parbodh, A.J.McDonald, and others, Soil and Tillage Research 199, 104595 (2020)

13. Lu Yahai, Xia Xingxuan, Gao Kailin, Huang Junjie, Ma Ke, Soil Biology and Biochemistry 143, 107740 (2020)

14. S. Radhika, K. Dolly, Renewable Energy 145, 682-690 (2020)

15. N. Halder Gopinath, D. Deepshikha, Environmental Science and Pollution Research 27(2), 1533-1557 (2020)

16. H. Mooyoung, H. Shervin, B. Siamak, Ecotoxicology and Environmental Safety 185, 109667 (2019)

17. K. Aggarwal Ashok, Y. Kuldeep, K. Nisha, Ja. Esha, International Journal of Recycling of Organic Waste in Agriculture 8, 21-26 (2019)

18. S. Yaspal, M. Vinay Kumar, A. Sanjay, G. Ravindra Kumar, D. Himanshu, Communications in Soil Science and Plant Analysis 50, 2640-2654 (2019) 